\title{
CAMPO Y DISTRIBUCIÓN: SIGNOS IDEOLÓGICOS E INICIATIVA DISCURSIVA EN LA POLÉMICA POR LOS IMPUESTOS A LA EXPORTACIÓN AGROPECUARIA EN LA ARGENTINA (AÑO 2008)
}

\author{
ALEJANDRA GABRIELA PALMA \\ Universidad Nacional del Sur, Bahía Blanca \\ CONICET-Centro de Estudios Lingüisticos "Dra. M. B. Fontanella de Weinberg"
}

\section{RESUMEN}

El presente artículo estudia la polémica discursiva suscitada en torno a las retenciones impositivas a las exportaciones agropecuarias que se desarrolló en Argentina en el año 2008. En este contexto, la presencia de determinados signos ideológicos (Voloshinov, 1976 [1926]), que se vuelven foco de lucha por la imposición de distintas acentuaciones, es un indicador importante de la construcción de la polémica discursiva. Nuestro propósito es analizar la circulación de los signos campo y distribución con el objetivo de detectar su rol en la lucha por establecer una iniciativa discursiva (Raiter et al., 1999). Para tales fines investigamos, de manera contrastiva, en discursos públicos emitidos por la Presidenta argentina, por un lado, y por dirigentes agrarios opuestos a la medida, por otro, las acentuaciones que adquieren estos dos signos. En primer lugar, se analizan las relaciones léxico-semánticas puestas en juego (Dubois, 1962), así como el rol de estos signos en la configuración de distintos tópicos (Van Dijk, 1978) que organizan los significados locales de estos discursos. Asimismo, a fin de especificar las diversas maneras en que se construyen estos tópicos, consideramos los componentes del discurso político (Verón, 1984), así como la presencia de formas de heterogeneidad mostrada (Authier-Revuz, 1984) en el encadenamiento discursivo e interdiscursivo de tópicos. El análisis revela la complejidad de uno de los posibles nodos de esta polémica: el rol del Estado en la generación de crecimiento económico y de la distribución equitativa de sus efectos.

PALABRAS CLAVE: discurso politico, polémica discursiva, conflicto agrario argentino 2008

\section{ABSTRACT}

This article aims to study the discursive polemic regarding the proposed new schema of agro-export taxes that took place in Argentina during the first half of the year 2008. In this context, the presence of ideological signs (Voloshinov, 1976 
[1926]), that are the focus of discursive struggles for the imposition of different accentuations, becomes an important indicator of the construction of the discursive polemic. The purpose of this paper is to analyze the circulation of the signs campo and distribución, in particular, with the objective of determining their role in the struggle to establish discursive iniciative (Raiter et al., 1999) within said polemic. To that end, we analyze and contrast, in public speeches of the argentinean President, on the one hand, and of leaders of the agrarian organizations oposed to the new tax, on the other, the acentuations that these two signs acquire. In the first place, we consider the lexical-semantic relations at play (Dubois, 1962), as well as the role of these signs in the configuration of different topics (Van Dijk, 1978) that organize these discourses' local meanings. Furthermore, in order to specify the various ways in which these topics are constructed, we also take into account the components of political discourse defined by Verón (1984), as well as the presence of forms of manifest heterogeneity (Authier-Revuz, 1984) in the discursive and interdiscursive linkage of topics. The analysis reveals the complexity of one of the possible nodes of this polemic: the role of the State in the generation of economic growth and the equitable distribution of its effects.

KEYWORDS: political discourse, discursive polemic, Argentinean agrarian conflict 2008.

\section{INTRODUCCIÓN}

Este trabajo forma parte de una investigación sobre discurso político mediatizado (Fairclough, 1995), centrada en el estudio de la polémica discursiva surgida en torno al establecimiento de un nuevo esquema de retenciones móviles -impuestos por derechos a la exportación de los bienes agropecuarios- en Argentina entre marzo y julio del año $2008^{1}$.

El denominado "conflicto del campo" tuvo como punto de inicio el 11 de marzo de 2008, con el anuncio por parte del Gobierno Nacional de la Resolución Ministerial 125 (en adelante, R125), que establecía un aumento de las retenciones agropecuarias a las exportaciones de soja y girasol, implantando un sistema móvil para las mismas, según el cual el porcentaje a retener subiría proporcionalmente al aumento del precio internacional de dichos productos. Como reacción frente a esta medida, las cuatro organizaciones que reúnen al sector empleador de la producción agroganadera en la Argentina -la Federación Agraria Argentina (FAA), la Confederación Intercooperativa Agropecuaria Limitada (CONINAGRO), la Sociedad Rural

${ }^{1}$ Esta investigación se lleva a cabo en el marco de una Beca Doctoral del Consejo Nacional de Investigaciones Científicas y Técnicas (CONICET), dirigida por los Doctores Elizabeth M. Rigatuso (UNS, CONICET, Academia Argentina de Letras) y Alejandro Raiter (Universidad de Buenos Aires). Se enmarca a su vez en el Proyecto de Grupo de Investigación "Estilo(s) comunicativo(s) en la interacción verbal del español bonaerense: construcción de identidades, valores y creencias", dirigido por la Doctora Elizabeth Rigatuso, subsidiado por la Secretaría General de Ciencia y Tecnología de la UNS. 
Argentina (SRA) y las Confederaciones Rurales Argentinas (CRA) - declararon el 12 de marzo un cierre patronal. La medida, que se extendió por 129 días, dio lugar, según Eduardo Sartelli, a uno de los más largos e importantes conflictos agrarios de la historia argentina, iniciándose como un enfrentamiento por reivindicaciones económicas para luego desembocar en una crisis política (Sartelli, 2008: 176).

Durante este tiempo, la Mesa de Enlace de Entidades Agropecuarias, compuesta por las cuatro entidades patronales mencionadas, coordinó, a través de los líderes de dichas organizaciones, una serie de medidas destinadas a interrumpir las actividades de comercialización de granos, así como el transporte interurbano y las exportaciones agrarias, realizándose cierres patronales parciales y bloqueos de rutas y puertos, a los que se sumaron acciones de protesta, como asambleas y movilizaciones de productores agropecuarios. Posteriormente, la situación se agravó por una huelga de los empresarios transportistas que incluyó bloqueo de rutas, lo cual empeoró aún más el abastecimiento de las ciudades.

Ante la extensión del conflicto en el tiempo, el 17 de junio la Presidenta Cristina Fernández de Kirchner envió un proyecto de ley al Congreso de la Nación, para ratificar la R125 y ampliar la legitimidad de la medida. El proyecto especificaba algunas cuestiones, en especial al fijar el monto del impuesto a las exportaciones de granos según una escala. Finalmente, un mes después, el 17 de julio, la Cámara de Senadores rechazó el proyecto de ley enviado por el Poder Ejecutivo, en una votación que debió ser desempatada por el Vicepresidente, Julio Cobos. Al día siguiente, el Poder Ejecutivo anunció la derogación de la R125.

En este marco, a fines de detectar de qué manera y bajo qué tópicos se organiza la polémica discursiva entre el Gobierno Nacional y los representantes de las organizaciones patronales que se opusieron a la R125, resulta productivo centrarse en la circulación de determinados signos ideológicos, que se vuelven foco de lucha por la imposición de distintos valores (Voloshinov, 1976 [1926]). En esta oportunidad, el análisis se enfoca en dos signos particularmente significativos, campo y distribución, en discursos públicos emitidos por la Presidenta Cristina Fernández de Kirchner (en adelante CFK), por un lado, y por los dirigentes de las organizaciones patronales agrarias, por otro.

\section{MARCO TEÓRICO-MetOdOLÓGICO}

1.1. Desde la perspectiva del análisis crítico del discurso, la investigación parte de la noción de discurso político mediatizado propuesta por Fairclough (1995), quien lo define como un orden del discurso consti- 
tuido por la compleja articulación de elementos de los órdenes del discurso del sistema político y del orden del discurso periodístico. Nuestro objetivo principal es detectar el lugar de los signos campo y distribución dentro de la lucha por establecer una iniciativa discursiva (Raiter et al., 1999) en el marco de la coyuntura (Chouliaraki y Fairclough, 2001) constituida por el debate público en torno a la modificación del régimen de las retenciones agropecuarias.

Por una parte, se intentará determinar los valores que adquieren los signos seleccionados en contextos de ocurrencia específicos, a través de las relaciones sintagmáticas que establecen con otros signos de la cadena (Dubois, 1962). En segundo lugar, se tomarán en cuenta las formas de heterogeneidad mostrada (Authier-Revuz, 1984) que, en estos contextos específicos, puedan dar cuenta de marcas de iniciativa discursiva en la lucha por la imposición de distintos sentidos. Así, la perspectiva teóricometodológica adoptada integra distintos niveles de análisis: léxico-semántico, semántico-textual, discursivo e interdiscursivo.

En cuanto al nivel léxico-semántico, resulta clave para el estudio de polémicas públicas la noción de signo ideológico propuesta por Voloshinov (1976 [1926]), quien postula que los signos lingüísticos empleados por los hablantes de una determinada comunidad cobran un alcance o valor social particular por su utilización, y, en tanto son ideológicos, pueden recibir distinta orientación. Por lo tanto, la lucha por los distintos acentos que puede llevar un signo constituye parte de una polémica discursiva. De esta manera, llamamos signo ideológico a un lexema o conjunto de lexemas que, dentro de una producción discursiva dada, reaparece con frecuencia y se vuelve relevante para otorgarle una significación determinada (Raiter et al., 1999). En este punto, para detectar los valores que adquieren los signos campo y distribución en estos discursos, se considerarán las relaciones de identidad, asociación y oposición (Dubois, 1962) que, en contextos específicos, se establecen entre los lexemas estudiados y otros signos presentes en la cadena.

En segundo lugar, en el nivel semántico-textual se relevará la configuración de tópicos (Van Dijk, 1978; Pérez, 1999; Raiter, 2014), entendidos como unidades de información semántica que organizan los significados locales del discurso (Van Dijk, 1978), en la articulación de los cuales se volverán relevantes las relaciones léxico-semánticas señaladas arriba. En este aspecto, centraremos la exposición en el rol que cumplen los signos campo y distribución en la construcción de estos tópicos, en especial a través de las relaciones que establecen con otros signos presentes.

Consideramos que los tópicos, en tanto unidades de información semántica, constituyen elementos discursivos, dado que los significados surgen necesariamente de las condiciones de producción de los discursos y 
las relaciones complejas que entre ellos se establecen. El trabajo con esta unidad de análisis en una serie de textos puestos en diálogo permite describir la complejidad del desarrollo de una polémica discursiva y las relaciones que pueden establecerse entre los distintos focos de disputa.

De esta manera, en el nivel discursivo, los componentes del discurso político señalados por Verón (1987) resultan conceptos operativos para relevar las distintas orientaciones discursivas que presentan los tópicos. Verón distingue dentro del discurso político cuatro componentes o zonas del discurso que definen las modalidades a través de las cuales el enunciador construye su red de relaciones con las entidades del imaginario: componente descriptivo, del orden de la constatación, que implica una lectura del pasado y de la situación actual; componente didáctico, que se relaciona con la enunciación de principios generales; componente prescriptivo, perteneciente al orden de la necesidad deóntica; y componente programático, del orden del poder hacer, que implica una orientación al futuro en el planteamiento de un programa o plan de acción.

Por otra parte, entendemos que, en el marco de un debate público, el intento por establecer la iniciativa discursiva se relaciona con el otorgamiento de distintos valores para los signos que son focos de la polémica. Concebimos este proceso en términos de paradigmas de disputa (Trew, 1983), donde los distintos valores que se pretenden imponer a los signos en una disputa discursiva corresponden a posicionamientos ideológicos en pugna.

Finalmente, consideramos adecuado adoptar también una mirada interdiscursiva. Dado que todo discurso está inmerso en una red que lo precede (Foucault, 1971), los signos ideológicos son focos de luchas por el establecimiento de valores o acentuaciones. Centrar el análisis en esta pugna discursiva exige no perder de vista que la construcción de la significación de un discurso está dada necesariamente por el lugar que ocupa-puede ocupar- en la red de discursos que lo preceden (Raiter, 2009). Siguiendo a Pêcheux (1988 [1975]) y Authier-Revuz (1984), llamamos interdiscurso a este exterior constitutivo del discurso.

En este sentido, resulta pertinente, al estudiar el encadenamiento discursivo e interdiscursivo de tópicos y signos dentro del contexto de una polémica pública acotada, analizar las formas de heterogeneidad mostrada (Authier-Revuz, 1984), en tanto formas lingüísticas que representan diversos modos de negociación del sujeto hablante con la heterogeneidad constitutiva de su discurso. Por un lado, estas formas, al designar un exterior con el cual se marca distancia, intervienen en el proceso por delimitar e imponer determinados valores a los signos en el interior de los discursos. Por otro lado, a causa del tipo de relación con ese otro que se designa, se vuelven claves en la construcción de una toma de posición, aspecto constitutivo del enfrentamiento polémico. 
Incluiremos entonces en nuestro análisis la presencia de formas marcadas de heterogeneidad mostrada, que indican que un fragmento tiene un estatuto diferente en el hilo del discurso. Principalmente, estas marcas son: discurso referido, donde un fragmento es mencionado como externo, y formas de connotación autonímica, que implican que la palabra o fragmento con estatuto diferente son a la vez mencionados y utilizados (AuthierRevuz, 1984). Incorporamos en esta última categoría a la negación polémica, que mantiene necesariamente una relación de contradicción con el enunciado que refuta (Maingueneau, 1994). En casos específicos, se hará referencia también a formas no marcadas de heterogeneidad mostrada (discurso indirecto libre, ironía, etc.), en las que se establece una distancia con un elemento del discurso sin designar su procedencia externa con una marca unívoca (Authier-Revuz, 1984).

En cuanto al procesamiento y elaboración de los datos, se ha realizado un cómputo con el software Wordsmith, restringiendo manualmente la búsqueda de material léxico al sustantivo campo en singular, referido al sector agrario de la economía y/o a sus actores ${ }^{2}$, y al sustantivo distribución, en su acepción de 'distribución del ingreso', así como sus formas verbales y adjetivas. Asimismo, se han incluido todas las formas léxicas de distribución acompañadas por el prefijo $r e^{-}{ }^{4}$.

1.2. El corpus de análisis está compuesto por los principales discursos públicos producidos en el marco de la coyuntura seleccionada, el debate en torno a las retenciones móviles. Se trata de discursos orales ${ }^{5}$ emitidos por la Presidenta Cristina Fernández de Kirchner y los representantes de las

${ }^{2}$ Este uso es destacado por Aronskind (2014), quien consigna que la utilización de campo para hacer referencia a los productores agropecuarios organizados se remonta a principios del siglo Xx, destacando como fuente documentos de la Sociedad Rural Argentina (SRA). Esto implica un pasaje de la referencia geográfica a la referencia social en el uso de este vocablo. Por otra parte, señala que, durante el conflicto agrario del año 2008, su uso se transforma para designar, en la prensa, a la Mesa de Enlace de Entidades Agropecuarias en expresiones como "la protesta del campo", por ejemplo. Ver también, con respecto a esta utilización del lexema campo durante la coyuntura estudiada, Palma (2011), donde se ha relevado el efecto homogeneizador de su uso por parte de Alfredo De Angeli (FAA Entre Ríos) en entrevistas televisivas y discursos públicos de diversa índole, y Palma (2013), donde se estudian, en artículos publicados en la prensa gráfica durante las primeras dos semanas del conflicto, los lexemas relacionados con la construcción de la polémica, donde el signo campo resulta clave (relevado también en expresiones como "la protesta del campo" y "el paro del campo").

3 Término que proviene del ámbito disciplinar de las finanzas públicas y es utilizado en el diseño de políticas públicas (cfr. Gasparini, 2006).

${ }^{4}$ Para referirnos a ellas en su conjunto usamos la forma nominalizada distribución por ser la más frecuente (36 sobre 53 en total).

${ }^{5}$ El carácter oral de estos discursos se ve reforzado por el hecho de que los emisores no leen escritos elaborados previamente, sino que pronuncian estos discursos ante su audiencia. 
organizaciones patronales que conformaron la Mesa de Enlace de Entidades Agropecuarias: Eduardo Buzzi (FAA), Mario Llambías (CRA), Fernando Gioino (CONINAGRO), Luciano Miguens (SRA), y Alfredo de Angeli (FAA Entre Ríos). En todos los casos, fueron pronunciados ante el público presente en diversas manifestaciones y retransmitidos por los medios de modo masivo.

En el caso de los discursos presidenciales, se han seleccionado aquellos discursos públicos en los cuales se hace referencia central y explícita al conflicto agrario. Se trata de siete discursos, cuatro emitidos desde la Casa de Gobierno (25 de marzo, 31 de marzo, 9 de junio y 17 de junio de 2008) y tres pronunciados en el marco de actos públicos de convocatoria masiva organizados en apoyo a la medida y al gobierno nacional (27 de marzo, 1 de abril y 18 de junio de 2008). Estos discursos fueron transmitidos por canales de noticias de alcance nacional, y tres de ellos fueron difundidos a través de la cadena nacional. Es el caso de los discursos emitidos el 9 de junio, donde se anuncia el "Programa de Redistribución Social"; el 17 de junio, cuando CFK comunica desde la Casa de Gobierno el envío del proyecto de ley sobre retenciones el Congreso de la Nación; y el 18 de junio, en el marco de una movilización en apoyo al Gobierno ${ }^{6}$.

Con respecto a los discursos emitidos por los representantes de las cuatro entidades agropecuarias que convocaron y lideraron las medidas de protesta, se han seleccionado aquellos pronunciados durante las dos movilizaciones más grandes realizadas en el marco del conflicto, en razón a su alto impacto mediático. La primera fue llevada a cabo en la ciudad de Rosario el día 25 de mayo de 2008, donde, en el marco de la fiesta patria ligada a la llamada Revolución de Mayo, se nuclearon alrededor de 250.000 personas ${ }^{7}$. La segunda, de una convocatoria similar, se realizó en Palermo,

\footnotetext{
${ }^{6}$ Se ha accedido al registro audiovisual de estos discursos en la página oficial de la Presidencia de la Nación (<http://www.casarosada.gob.ar/>) y en distintos portales electrónicos.

7 El lugar y el momento elegidos agregan significaciones importantes a la manifestación. En primer lugar, la realización del acto en la ciudad de Rosario, cabecera de un importante distrito agrícola y centro del gran complejo agroindustrial y portuario de la soja, refuerza por un lado la vinculación con los productores del interior del país, en especial de las regiones pampeana y litoral de la Argentina, líderes en producción de soja, girasol y trigo. Por otra parte, enfatiza el carácter "federal" del acto (en oposición al "centralismo" del Gobierno Nacional, representado por la Ciudad Autónoma de Buenos Aires) (Sartelli, 2008). La elección de la fecha, 25 de mayo, al mismo tiempo que acentuó su carácter simbólico, al coincidir con un festejo patrio de larga tradición de celebración, convirtió a la manifestación de las organizaciones agrarias en un acto paralelo al que tradicionalmente se desarrolla de manera oficial. En esta oportunidad, el acto oficial, presidido por CFK, fue llevado a cabo en la provincia de Salta, ubicada en el Norte del país, alejado de los principales focos del conflicto. En su discurso, la Presidenta no realizó referencias explícitas al conflicto, centrándose en la conmemoración de la fecha patria y de los hechos y personajes históricos vinculados a ella.
} 
el principal espacio abierto de la Ciudad Autónoma de Buenos Aires, el 15 de julio, ante la eminente votación del proyecto sobre retenciones móviles en el Senado de la Nación. Dada la importancia que los medios de comunicación nacionales le dedicaron al conflicto, ambas manifestaciones, y en especial las alocuciones de los dirigentes, fueron transmitidas en directo por los canales de noticias $T N$ y Canal Rural, y retransmitidas parcialmente en los noticieros vespertinos ${ }^{8}$.

Los discursos seleccionados pueden ser clasificados dentro de lo que Raiter (2009) denomina discurso público político. Este tipo de discurso se caracteriza por:

a) ser producido en un evento comunicativo público, donde un emisor responsable, que tiene un rol social aceptado como líder (de una agrupación u organización, o de una protesta social espontánea), se dirige a los otros numerosos participantes, que toman el rol de audiencia presente, al mismo tiempo que pueden identificarse con actores sociales que apoyan a ese líder,

b) tener como fin "producir un cambio y/o confirmación de conductas, creencias y actitudes a partir de la presentación de una pararrealidad discursiva”. (Raiter, 2009: 76)

\section{ANÁLISIS}

Como hemos señalado, el foco de análisis está constituido por los signos campo y distribución, y, en especial, a través de la relación que establecen entre sí y con otros lexemas relevantes en contextos específicos, el lugar que ocupan en la configuración y encadenamiento de distintos tópicos. De esta manera, un análisis de conjunto de los contextos de ocurrencia de los signos campo y distribución revela algunas tendencias que intentaremos especificar tomando algunos casos especialmente representativos en relación con los objetivos propuestos.

Los signos campo y distribución, en tanto focos de distintas valoraciones, están constituidos por lexemas que ocurren en contextos específicos de aparición. En primer lugar, el lexema campo, constitutivo del signo campo en estos discursos, aparece en singular haciendo referencia al sector agrario de la economía y/o a sus actores 67 veces en los discursos pronunciados por los dirigentes agrarios y 26 veces dentro de los discursos públicos de CFK. En segundo lugar, el signo distribución en su acepción de 'distribu-

${ }^{8}$ Se ha accedido al material audiovisual a través del sitio del Canal TN (<http://tn.com.ar/ videos $>$ ) y diversos portales electrónicos (en especial <www.youtube.com/arribacampo >). 
ción del ingreso' se encuentra constituido en estos discursos por distintos lexemas: distribución, distribuir, distributivo/a, por un lado, y las formas acompañadas por el prefijo re, por el otro. En conjunto, se detectaron 44 ocurrencias en los discursos pronunciados por CFK frente a 9 dentro de los discursos emitidos en las manifestaciones agrarias del 25 de mayo y el 15 de julio.

Tal como se adelantó, limitaremos la exposición a la descripción de los valores que adquieren estos dos signos en el marco de la construcción y articulación de tópicos recurrentes en los dos grupos de discursos analizados.

Por una parte, en lo que respecta a los discursos producidos en las manifestaciones agrarias, hemos relevado en trabajos anteriores la recurrencia de un tópico en especial: (MA1) "la economía argentina se centra principalmente en la producción de materias primas", que promulga el modelo económico agroexportador como la mejor opción para la Argentina (Palma, 2014). A su vez, encontramos que este tópico puede vincularse con otro, ligado a lo que en política económica se denomina teoría del derrame económico (Gaviola y Anchorena, 2008): (MA2) "el crecimiento económico del sector agropecuario genera crecimiento en otros sectores".

Por otra parte, en los discursos emitidos por CFK en la coyuntura seleccionada, cobran relevancia, en relación a la asignación de valores para los signos campo y distribución, dos tópicos en particular: (CF1) "el modelo económico instalado a partir de 2003 por Néstor y Cristina Kirchner generó las condiciones para la recuperación económica del país luego de la crisis producida por el modelo neoliberal”, y (CF2) "para seguir avanzando hacia una sociedad más equitativa, es necesario distribuir el excedente generado".

\subsection{Campo}

En primer lugar, centramos el análisis en el signo campo en los dos grupos de discursos estudiados. Comenzaremos por el caso de los discursos de los dirigentes agrarios, donde este signo presenta mayor frecuencia, para luego contrastar los resultados con lo relevado en los discursos de CFK.

En trabajos anteriores, referidos a la construcción de destinatarios en los discursos de los dirigentes agrarios durante la polémica señalada (Palma, 2014), hemos remarcado que la utilización del signo campo como colectivo (Adelstein y Vommaro, 2014) puede relacionarse con una estrategia común a ellos. Al hacer referencia conjunta al sector agrario de la economía y a sus actores sociales, este uso genera un efecto entre ambiguo y homogeneizador, en tanto desfocaliza la complejidad económica y social en juego. 
En esta oportunidad, nos interesa explorar, en este mismo grupo de discursos, de qué manera el signo campo se enmarca en los tópicos ya señalados referidos al rol del sector agropecuario en el funcionamiento de la economía argentina. En especial, encontramos que la articulación de los tópicos MA1 (Argentina como país productor de materias primas) y MA2 (la actividad agropecuaria genera crecimiento en toda la economía) puede apreciarse en parte en la relación presente entre los signos campo y producción.

Destacamos en este marco dos fragmentos en particular, correspondientes a discursos pronunciados por Fernando Gioino (CONINAGRO) el 25 de mayo en Rosario y por Luciano Miguens (SRA) en el acto público realizado el 15 de julio en la ciudad de Buenos Aires, respectivamente, donde la vinculación entre estos dos signos puede observarse en las asociaciones que se establecen entre los lexemas que los constituyen (produciendo, producir, producción y campo).

(1) NO QUEREMOS (0.5) VOLVER A REPETIR, (0.5) QUEREMOS NO ENFRIAR LA ECONOMÍA, (0.5) Y SEGUIR PRODUCIENDO MÁS. (.) y hoy tenemos un desafío. (0.5) sí señores, (1) tenemos un desafio muy grande, (1) el que nos dan estos tiempos. (1) el campo ha demostrado que puede producir más, (x) puede producir el doble o mucho más. (1) un mundo que nos demanda, (1) tenemos que aprovechar esta oportunidad. (0.5) tenemos posibilidad de darle COMIDA en cantidad y calidad para todos los argentinos, (0.5) y exportar con muchos beneficios para todo el país el resto. (Fernando Gioino, 25 de mayo de 2008) ${ }^{9}$

(2) la Argentina tiene una gran oportunidad histórica de la mano del campo. (1) para aprovecharla, (0.7) hay que tener un proyecto político consensuado. (1) no ayudaría una ley (1.5) que atente contra la producción, (1) desaliente la inversión, (1.5) afecte a las economías (x) provinciales (1) y genere desempleo en todo el país. (1) en los últimos años (1) el campo ha generado una verdadera revolución tecnológica, (0.8) con fuerte aporte de capital, (1) y creación de numerosos puestos de trabajo. (1) este ha sido un proceso silencioso, (1) pero palpable y consistente, (1) y la unánime reacción de la gente (1) marca la férrea voluntad de preservarlo. (Luciano Miguens, 15 de julio de 2008)

En ambos fragmentos se observa una coincidencia en la presentación de los dos tópicos, mediante una asociación entre campo y producción y la personificación de campo ("el campo ha demostrado...", "el campo ha ge-

${ }^{9}$ La transcripción de los datos se realizó según las convenciones de transcripción incluidas en Van Dijk (2000), ideadas originalmente por Gail Jefferson. Se presenta un anexo que detalla los signos y caracteres empleados. Las cursivas se han introducido para destacar las voces claves en los ejemplos. 
nerado...”). Asimismo, se destaca en ambos casos la asociación campooportunidad, que subraya un movimiento discursivo común que implica una proyección hacia el futuro, donde se encuentra implícita la idea de crecimiento económico $^{10}$. Sin embargo, las diferencias que presentan dan cuenta de dos movimientos discursivos distintos en la articulación de los dos tópicos.

En el caso de Fernando Gioino (ejemplo 1), la utilización del lexema campo es indicativa del rol privilegiado que se le asigna al sector agropecuario en la dinámica económica. Esto se remarca mediante una secuencia temporal orientada hacia el futuro, subrayada por diversos elementos ponderativos ligados al signo producir: "seguir produciendo", "puede producir el doble o mucho más”. Cobra relevancia aquí el componente programático (Verón, 1987), reforzado también en los signos desafí y oportunidad. En este marco, el tópico MA2 (el crecimiento en el sector agropecuario genera efectos positivos en toda la economía), expresado como una evidencia atemporal -marcada por el uso del tiempo presente-, está subordinado al tópico ME1, relacionado con la producción de materias primas. Se restringe el sentido de producir a la actividad primaria -ligada a los alimentos-, que generará "beneficios" a través de las ganancias provenientes de las exportaciones, presentando a la sociedad en su conjunto como beneficiaria de los procesos llevados a cabo por este actor (hecho subrayado por la utilización del adjetivo todo en ambos casos: "para todos los argentinos", "para todo el país").

En el fragmento perteneciente al discurso de Luciano Miguens (ejemplo 2), los efectos positivos de la actividad agropecuaria, ligados al crecimiento económico, están presentes en la asociación campo-oportunidad, y son a la vez explicitados indirectamente en la presentación negativa de la medida adoptada por el Gobierno Nacional y la posibilidad de que el proyecto de ley sobre los impuestos a la exportación sea aprobada por el Senado de la Nación. A la vez, el tópico del crecimiento concatenado (MA2) se desarrolla desde una descripción de una recuperación económica donde, nuevamente, el agente es el colectivo campo. A diferencia de la proyección hacia el futuro que encontramos en el ejemplo 1, en este caso el eje parece ser el componente descriptivo (Verón, 1987). Se presenta un estado de cosas desde signos que hacen referencia a procesos materiales relacionados con las actividades productivas y sus efectos: generación, creación, aporte, proceso palpable y consistente e incluso revolución tecnológica no son proyectos sino hechos consumados. De esta manera, no se pone el acento

\footnotetext{
${ }^{10}$ Para una expresión de la relación léxico-semántica entre campo y crecimiento en este conjunto de discursos, véase infra el ejemplo 8, perteneciente al discurso de Fernando Gionio en el acto del 25 de mayo de 2008 .
} 
en la profundización de un cambio sino en la preservación del modelo vigente. Esta necesidad de conservación surge a raíz de una amenaza externa (el proyecto de retenciones móviles enviado al Congreso), y se refuerza en la oposición suscitada entre los lexemas valorados negativamente-desaliente, afecte y desempleo-, vinculados al proyecto de retenciones, y los lexemas valorados positivamente -campo, generación, creación y aporte, con clave en revolución tecnológica-, referidos al crecimiento del sector y sus efectos sobre toda la economía. Por otra parte, si bien estos efectos positivos son descriptos como evidencias, los signos cargados positivamente destacan la idea de un crecimiento dinámico que contrasta con el estancamiento asociado al proyecto de ley. Así, la oposición presentada refuerza el tópico del crecimiento concatenado (MA2): la oportunidad de crecimiento desde el sector agropecuario presupone un Estado que no intervenga, que deje fluir el "derrame económico".

Estas dos direcciones, la proyección hacia el futuro desde una idea de avance y la valorización conservadora de un estado de cosas actual, que pueden rastrearse a lo largo de los discursos de los dirigentes agrarios, coinciden en lo que resulta nodal a la relación entre los dos tópicos: el rol privilegiado que se le da al sector agropecuario en el funcionamiento de la economía nacional. Este rol se expresa de manera diversa en la articulación de dichos tópicos. Por un lado, el tópico MA1 ("la economía argentina se centra principalmente en la producción de materias primas"), pone al sector agropecuario en el centro del proceso de crecimiento económico, atravesado por distintas dimensiones temporales. Por otro, mediante el tópico MA2 ("el crecimiento económico del sector agropecuario genera crecimiento en otros sectores”), se destaca que este proceso se da de manera espontánea -y con el campo como "motor" impulsor-, por lo cual el Estado no debe perturbarlo.

Como hemos señalado, analizaremos ahora el valor que adquiere el signo campo en los discursos de CFK en el marco de la polémica, para lo cual nos centramos en la relación que se establece entre los dos tópicos mencionados: (CF1) "el modelo económico instalado a partir de 2003 por Néstor y Cristina Kirchner generó las condiciones para la recuperación económica del país luego de la crisis producida por el modelo neoliberal", y (CF2) "para seguir avanzando hacia una sociedad más equitativa, es necesario distribuir el excedente generado". La articulación de estos dos tópicos se expresa en un movimiento que va desde la presentación de la recuperación económica como evidencia (componente descriptivo) a la proyección hacia el futuro (componente programático, Verón, 1987), que implica una explicitación de los pasos a seguir. Este movimiento puede apreciarse en dos fragmentos pertenecientes al discurso pronunciado el 27 de marzo: 
(3) con la legitimidad que nos da que en este proyecto económico, social, político y cultural. (1) han crecido todos, (1) ha crecido el campo, ha crecido la industria, (0.5) los trabajadores han recuperado el trabajo perdido, el salario, (2) estamos recuperando de vuelta un lugar en la tierra, argentinos, (1.5) un lugar en el mundo, nuestro lugar (1) la república argentina, (2) en nombre de esas legitimidades, (1) la del pueblo, (1) la del resultado económico y, fundamentalmente, (1) para que todos los argentinos y para que todas las argentinas (0.5) podamos vivir mejor, (1) los convoco al diálogo en serio, desde aquí. (Cristina Fernández de Kirchner, 27 de marzo de 2008)

(4) quiero decirles que no es una política antisoja, (1) es una política esencialmente (0.5) pro argentina, pro pueblo, (1) pro campo también, pro pueblo. ((aplausos) ) no es una cuestión (1) como alguien quiere hacerlo aparecer (1) entre campo e industria. (0.5) una parte importante de la planta industrial es de la agroindustria. (2) todos son necesarios, el campo, la industria, (0.5) el comercio, los servicios. (1) pero todos tenemos que entender (0.5) que todos tenemos que participar armónicamente de ese crecimiento (0.5) porque es la única manera de erradicar (0.5) definitivamente el hambre y la miseria (0.5) en la Argentina (1.5) como lo estamos haciendo. (Cristina Fernández de Kirchner, 27 de marzo de 2008)

En primer lugar, en el ejemplo 3 observamos que el tópico de la recuperación económica ( $\mathrm{CF} 1)$ se expresa en la reiteración de los lexemas crecer, crecimiento y recuperar. Aquí, el signo campo no ocupa el lugar de agente del crecimiento que se ha detectado en los discursos de los dirigentes agrarios. Por el contrario, campo se convierte en uno de los sectores favorecidos por este proceso de reactivación. La inclusión de este signo en la enumeración de los distintos beneficiarios del crecimiento, a la vez que pone el foco en el proceso mismo, asigna un lugar subordinado al sector agropecuario en el conjunto de la economía. Por otra parte, esta descripción de los logros del modelo económico propuesto por el gobierno cobra significado desde un pasado que se restituye, y se proyecta hacia el futuro en la enunciación de lo que falta: "para que [...] podamos vivir mejor". La idea de progreso (crecimiento, vivir mejor) contrasta con la evaluación negativa del pasado, expresada fundamentalmente en la oposición pérdida-recuperación, que en este caso se enfoca en el signo trabajo, valorado como punto esencial de la reactivación económica.

En el ejemplo 4, por otra parte, el componente programático se relaciona de una manera más directa con la necesidad de avanzar hacia una redistribución del ingreso (tópico CF2). La negación polémica y la glosa que la acompaña -"no es una cuestión como alguien quiere hacerlo aparecer entre campo e industria"- aparecen aquí como formas de heterogeneidad mostrada que señalan un exterior al discurso, el discurso de un otro 
que no se especifica. En este caso, parece tratarse de una postura que promulga la actividad agroexportadora como modelo económico autosuficiente, que podríamos relacionar, según lo expuesto anteriormente, con una expresión extrema del tópico de la Argentina como productora de materias primas ${ }^{11}$. Así, este lugar de alteridad, del que se marca distancia a la vez a través de la oposición presente en los prefijos anti y pro, opera como marco para delimitar una toma de posición: la propuesta de un programa ("erradicar definitivamente el hambre y la miseria"), donde la expresión "como lo estamos haciendo" remite directamente a la justificación de la medida adoptada sobre las retenciones móviles. Por otra parte, la construcción concesiva "pro campo también", así como la inclusión de campo en una enumeración -similar a la presente en el ejemplo 3-, vuelve a colocar al colectivo campo como un elemento más entre otros en la descripción del funcionamiento económico. A su vez, la presencia de los lexemas todos, participar y armónicamente subraya la necesidad de un acuerdo (componente prescriptivo, Verón, 1987) para llevar adelante el proyecto políticoeconómico que se está proponiendo, y parece mitigar así la imposición que presupone la intervención del Estado a través de políticas públicas.

En este punto, ha de tenerse en cuenta que el anuncio de la R125 había ocurrido apenas dos semanas antes de la primera manifestación en apoyo al gobierno en el marco del conflicto, el 27 de marzo, donde CFK pronuncia este discurso. El anuncio produjo, como se ha descripto, el rechazo casi inmediato de las organizaciones patronales agrarias. En este contexto, una de las principales críticas que se le hicieron a la medida fue el hecho de haber sido impulsada desde el Poder Ejecutivo, sin discusión previa. Así, uno de los ejes de la polémica que comenzaba a formarse fue si el Estado debía intervenir de esta manera en la economía. Es teniendo en cuenta este contexto que debe entenderse la utilización de algunos de los recursos discursivos relevados en el discurso presidencial, vinculados a justificar y legitimar la medida adoptada.

\subsection{Distribución}

Resta ahora analizar el valor que adquiere el signo distribución, en su acepción de 'distribución del ingreso', en los dos grupos de discursos estudiados. En este caso, por ser un signo con mayor presencia en el discurso de CFK, comenzaremos por el análisis de los discursos presidenciales, para luego contrastarlo con lo relevado en los discursos de los dirigentes agrarios.

${ }^{11}$ Esta postura extrema que promulga la actividad económica como autosuficiente en desmedro de la industrial no se ha relevado en los discursos de los dirigentes agrarios. 
En los discursos de CFK, la relación entre los dos tópicos estudiados -(CF1) recuperación económica de la mano del Gobierno y (CF2) necesidad de redistribuir el excedente generado- puede apreciarse a su vez en la asociación entre los signos distribución y rentabilidad. En cuanto a distribución, observamos que el valor del signo está relacionado con el componente prescriptivo (Verón, 1987), del nivel de la necesidad deóntica, y esta necesidad, a la vez, se construye mediante marcas de heterogeneidad mostrada, como previa al momento de enunciación. La necesidad de redistribuir el ingreso se introduce como un tópico ya presente en el interdiscurso $^{12}$, para luego redefinirlo al articularlo con el tópico de la recuperación a través de los componentes didáctico y programático.

Ilustraremos esta observación a través de dos fragmentos, uno extraído del primer discurso pronunciado por CFK durante la coyuntura del conflicto, el 25 de marzo, y otro perteneciente al discurso emitido el 9 de junio, en el marco del anuncio del envío del proyecto de retenciones móviles al Congreso.

En el caso del ejemplo 5, perteneciente al discurso pronunciado el 25 de marzo, la articulación de los dos tópicos estudiados se encuadra en una redefinición del concepto de retenciones móviles, con una marcada reiteración del signo distribución bajo distintas formas léxicas:

(5) esto es (1) ((aplausos) ) (2) lo que yo digo (4) lo que yo digo (2) ((aplausos)) qué país (1) queremos, si queremos volver al país (1) de unos poquitos (1) o queremos volver a un país (0.5) más justo, (1) con mayor equidad, (0.5) con mayor distribución. porque las retenciones, no son medidas fiscales, (1) son profundas medidas redistributivas del ingreso. escucho y leo (1) muchas veces $(0.5)$ a (0.5) periodistas (1) que (0.5) por allí (1) tienen un marcado tinte (1) progresista, encarar el tema de las retenciones, desde una percepción únicamente fiscal. pero (1) a ver, qué es la distribución del ingreso? cómo se hace la distribución del ingreso si no es, precisamente, sobre aquellos sectores que tienen rentas extraordinarias, si no de qué ejercicio me están hablando en materia de (x) distribución del ingreso? (Cristina Fernández de Kirchner, 25 de marzo de 2008)

$\mathrm{Al}$ comienzo del fragmento está presente nuevamente la idea de recuperación, en este caso con eje en el componente programático (Verón, 1987), es decir, proyectada hacia el futuro, donde se destaca una identificación léxica entre justicia, equidad y distribución ${ }^{13}$. Aquí se produce la redefi-

${ }^{12}$ La presencia de marcas discursivas explícitas que pueden interpretarse como referencias al interdiscurso es señalada por Raiter (2009) como una característica del discurso de CFK.

${ }^{13}$ Excede a los objetivos del presente estudio la exploración de las posibles relaciones interdiscursivas que se establecen en el discurso de CFK entre el signo distribución del ingreso y el signo justicia social, propio del imaginario peronista. 
nición, a través de la negación - "no son medidas fiscales"- y la posterior rectificación (García Negroni, 2009) -“son profundas medidas redistributivas del ingreso"-. Teniendo en cuenta lo ya expuesto, las "retenciones" son redefinidas como el punto intermedio entre el camino ya transitado (la recuperación, llevada a cabo mediante la reactivación económica) y los pasos dificultosos que faltan recorrer. Parte de esta dificultad reside en la pugna que está implícita en la construcción disyuntiva "volver al país de unos poquitos [...] o volver a un país más justo [...]", donde las dos opciones disponibles pueden leerse como la representación de lo que se considera el foco de la polémica en este discurso. En este contexto, el signo fiscal aparece como forma marcada de heterogeneidad mostrada, donde el lugar del otro se identifica: no se discute aquí con los adversarios políticos, sino con "periodistas" con "un marcado tinte progresista" que no han sabido interpretar lo sucedido. El contraste se destaca en la oposición léxica entre "medida fiscal" y "medida redistributiva", donde lo que subyace es una clasificación de la medida adoptada por el Gobierno. El tópico CF2, ligado a la redistribución del ingreso, aparece así encuadrado en una redefinición de la coyuntura misma, a través del componente didáctico, delimitado por el conector causal porque y por la pregunta retórica (Blas Arroyo, 2010) “¿qué es la distribución del ingreso?”. No se trata de un impuesto (solamente), sino de un "ejercicio" tendiente a restablecer un equilibrio social perdido. A la vez, la pregunta retórica "si no, ¿de qué ejercicio me están hablando en materia de distribución del ingreso?” refuerza la presentación del tópico CF2 como presente en el interdiscurso de manera independiente a la coyuntura del conflicto.

En el ejemplo 6, extraído del discurso del 9 de julio, se destaca la incorporación del signo pobreza a la relación secuencial entre los dos tópicos, (CF1) la recuperación económica -fuente de la rentabilidad del sector agropecuario-, y (CF2) la necesidad de distribuir el excedente generado, que relacionamos con la expresión anterior "erradicar el hambre y la miseria”. En el contexto del anuncio del envío del proyecto de retenciones móviles al Congreso de la Nación, CFK realiza una suerte de balance de la disputa política:

(6) la primera (1) cosa que se me ocurre, después de estos largos noventa días (1) es interrogarnos a nosotros, a todos, (1) a todos los argentinos, a los que tienen responsabilidades empresariales, a los que pertenecemos a los partidos políticos (1) y a todos los ciudadanos (0.7) si realmente estamos decididos a abordar en la república argentina (0.5) el problema de la pobreza. porque el problema de la pobreza (1) sin redistribución del ingreso y sin tocar rentas extraordinarias, compatriotas, es imposible. (Cristina Fernández de Kirchner, 9 de junio de 2008) 
Nuevamente, aquí las formas de heterogeneidad mostrada contribuyen a la delimitación de una escena desde la cual definir una propuesta. La presencia del signo pobreza en la construcción "la cuestión de la pobreza" demarca como una exigencia externa al hilo del discurso una preocupación que se considera activa en el interdiscurso con independencia al debate público por las retenciones móviles, hecho que es reforzado mediante el uso del adverbio realmente. En una operación similar a la analizada en el ejemplo 5, en el marco del componente didáctico -introducido también por el conector porque-, se redefine el valor del signo pobreza, y se lo vincula fuertemente con el tópico CF2 (la necesidad de redistribuir el excedente generado) a través de una negación polémica ("es imposible"). Por otra parte, en cuanto al componente programático, el valor del signo distribución se establece a través de su vinculación con los signos pobreza y rentabilidad. La introducción discursiva de signos ideológicos como presentes en el interdiscurso, vinculados a problemáticas sociales -distribución entre ellos-, subraya la operación de definición de valores propios para estos signos en relación con un programa de gobierno que viene a responder a esas demandas externas.

Tanto en (5) como en (6), la asociación entre el objetivo de distribuir el ingreso y las "rentas extraordinarias" del sector agropecuario es de dependencia: aquél no es posible sin éstas. El rol del Estado tiene que ver con "distribuir" un excedente, y su legitimidad para hacerlo se construye desde, al menos, dos lugares:

a) la afirmación de haber generado el modelo que hizo posible ese excedente, $y$

b) la supuesta circulación social del tópico CF2, su lugar de privilegio en la representación del interdiscurso en estos discursos.

Tal como se ha adelantado, este análisis del signo distribución en los discursos de CFK fue contrastado con el valor que adquiere este signo en los discursos de los dirigentes agrarios. En ellos, hemos relevado, en los contextos de aparición de distribución, marcas autonímicas que remiten a discursos favorables a la medida de retenciones móviles ${ }^{14}$. Tomamos en este

14 Para un análisis más extenso de la presencia en los discursos de los dirigentes agrarios del tópico de la (re)distribución del ingreso y su relación con la construcción del contradestinatario ver Palma (2014). Hemos detectado que la aparición de este tópico en estos discursos, si bien es generalmente atribuido al discurso presidencial mediante distintas marcas de heterogeneidad mostrada, no conlleva un cuestionamiento de su valor positivo: no se lo incluye explícitamente en la inversión de creencias, ni se plantea otro valor alternativo. Presentamos en el presente artículo dos fragmentos, uno representativo de esta tendencia y otro que presenta un aspecto complementario de la expresión de este tópico. 
caso un extracto del discurso pronunciado por Eduardo Buzzi en el acto público del 15 de julio, como caso representativo.

(7) DE QUÉ NOS HABLAN (1) ALGUNOS (0.5) INTERLOCUTORES (.) QUE VEMOS EN LOS MEDIOS (1) CUANDO NOS DICEN QUE SE IMPULSA (0.7) UNA REDISTRIBUCIÓN DESDE EL CAMPO (.) DESDE LA RENTA EXTRAORDINARIA QUE TIENE EL CAMPO, (1) CUANDO PARECE Y SE RECONOCE PÚBLICAMENTE (1) QUE EL AUMENTO DE RETENCIONES EN REALIDAD TIENE QUE VER (0.3) cOn (x) CON CUMPLIR LOS COMPROMISOS EXTERNOS? [...] Y POR QUÉ (1) NO SE PONE EL EJE EN AQUELLOS ACTORES GANADORES DE LOS NOVENTA, (0.5) QUE SIGUEN SIENDO LOS GANADORES (0.5) DE ESTE PRESENTE? ((aplausos)) POR QUÉ NO MIRAN (1) LO QUE PASA (.) POR EJEMPLO (0.5) CON LA RENTA FINANCIERA? (0.5) SE QUIERE (0.5) SEGUIR (x) SACANDOLE TIENTOS Y LONJAS (0.5) AL MISMO CUERO DE LOS CHACAREROS DEL CAMPO (0.5) Y DEL INTERIOR. (0.5) PERO NO SE GRA:VA LA RENTA FINANCIERA. (Eduardo Buzzi, 15 de julio de 2008)

Aquí, el signo distribución funciona como forma marcada de heterogeneidad mostrada: mediante el recurso del discurso referido indirecto (Authier-Revuz, 1984) se reproduce a través del lexema redistribución y el sintagma rentas extraordinarias, representativas de los signos distribución y rentabilidad, la posición de los actores mencionados de manera ambigua como "algunos interlocutores", expresión referencial caracterizada a través de una cláusula adjetiva, "que vemos en los medios", que no permite identificarlos con claridad. El discurso del adversario se presenta entonces a través de signos que hemos relevado como particularmente significativos en el discurso presidencial. Incluso se hace explícita la relación rentabilidaddistribución, y se incluye en ella el rol del sector agropecuario utilizando el signo $c a m p o^{15}$. Es interesante notar que, lejos de cuestionar el valor del signo distribución tal como lo hemos relevado en los discursos presidenciales, en este discurso se pone en duda, mediante una serie de preguntas retóricas, que ese sea el fin último de la medida tomada por el gobierno. Estas preguntas retóricas contienen una reorientación de los dos signos, distribución y rentabilidad, sin afectar su valor ni su relación: se presupone un acuerdo con respecto al tópico "es necesario distribuir el excedente generado", pero esa rentabilidad se despega del campo y se atribuye a otro sector, al modificar el sustantivo renta con el adjetivo financiera (combinación que no hemos encontrado en los discursos de CFK).

Nos interesa destacar, finalmente, un fragmento del discurso de Fernando Gioino del 15 de julio, donde se realiza una articulación entre el

${ }^{15}$ Obsérvese que en los ejemplos 5 y 6 , pertenecientes a discursos de CFK, el sintagma rentas extraordinarias aparece dos veces. No descartamos una interpretación irónica de esta reproducción discursiva por parte de Buzzi. 
tópico de la distribución del ingreso y lo que hemos denominado "efecto derrame", en una curiosa apropiación del signo distribución, que lo pone en relación con producción.

(8) evidentemente, (1) toda esa fuerza productiva, MÁS PRODUCTO, MÁs VALOR AGREGADO, (0.5) LA MÁQUINA DE LA RIQUEZA PRODUCIENDO NO LA PODEMOS PARAR. vamos a tener más trabajo, (0.5) más producción, (1) más integración de argentinos, (1) menos pobreza, (0.5) mejor distribución del ingreso, con equitativos (0.5) impuestos tributarios, (0.5) una equitativa distribución del ingreso (1) y lo que es más (0.7) un adecuado equilibrio social. (1.5) y llegamos acá, (0.5) pero llegamos bien, (1) llegamos contentos, (0.5) porque (1) este reclamo (1) llegó, (0.5) llegó (1) al congreso de la nación, [...] y la opinión ya está, (0.5) la importancia que tiene el campo, (0.5) y la posibilidad y la importancia que tiene la argentina de crecer TODOS JUNTOS junto al campo. (Fernando Gioino, 15 de julio de 2008)

Aquí la producción económica -a través de los signos producción, valor agregado, riqueza, trabajo- se presenta como solución para el problema de la desigualdad social, presente a través del lexema pobreza, y por oposición, en los signos integración, equitativa distribución, equilibrio social. En este caso la ausencia de marcas autonímicas en torno a la utilización del signo distribución resulta significativa. Sin embargo, entendemos que el lexema equitativo/a opera en este contexto como forma no marcada de heterogeneidad mostrada (Authier-Revuz, 1984), al estar presente en el interdiscurso como representativo del programa de gobierno de CFK en relación con la distribución del ingreso. Una marca de posible distancia con respecto al valor superador que se le asigna al signo distribución en el discurso de CFK -relacionado con la idea de eliminar la pobreza- es su presentación como insuficiente en relación con lo que se denomina "equilibrio social", cuyo valor superior está reforzado por la expresión modalizante intensificadora "lo que es más"16.

A la vez, el tópico de la distribución del ingreso se subordina significativamente a la idea de derrame económico, mediante una metáfora particularmente sugestiva: "la máquina de la riqueza produciendo no la podemos parar". Así, el efecto positivo de la actividad económica generada por el sector agropecuario es tan fuerte que se vuelve inevitable, absorbiendo la responsabilidad de generar equilibro -y, por lo tanto, alguna forma de distribución- en el proceso.

${ }^{16}$ En su clasificación de las estrategias de intensificación, Alabelda señala dentro de los procedimientos de intensificación del enunciado (es decir, de construcción de la responsabilidad por lo dicho) que "la combinación de ciertas categorías gramaticales en el discurso favorece la creación de un valor intensificador" (2005: 102). 
Finalmente, destacamos la presencia de la asociación campo-crecimiento, que, de manera similar a la observada anteriormente dentro de los discursos de los dirigentes agrarios entre campo y producción, es realizada a través de la idea de posibilidad, orientada hacia el futuro. Aquí, nuevamente, el componente programático parece ligarse al tópico del crecimiento concatenado (MA2), donde el sintagma "todos juntos" subraya el postulado de que el crecimiento se expandirá sobre la totalidad de los actores sociales, eliminando la necesidad de una intervención estatal para conseguir una "equitativa distribución del ingreso".

\section{Conclusiones}

El estudio de los signos campo y distribución en discursos considerados como centrales en el debate público en torno al establecimiento de un nuevo esquema de impuestos a los derechos de exportación agropecuaria en Argentina revela algunos de los sentidos que se ponen en juego, así como distintas articulaciones de tópicos que los enmarcan.

En primer lugar, en los discursos de los dirigentes agrarios, el signo campo tiene un lugar de privilegio en el encadenamiento de los tópicos presentados. El tópico de la caracterización de la Argentina como país productor de materias primas (MA1) aparece, bajo distintas formas, subordinado al tópico MA2, según el cual el crecimiento generado por el sector genera y generará espontáneamente las condiciones para un crecimiento sostenido que afecte positivamente a todos los sectores de la economía del país (proceso que hemos dado en llamar, en coincidencia con un concepto económico, "efecto derrame”). Así, la oposición a la medida adoptada por el Gobierno se expande, mediante la articulación de estos dos tópicos, más allá de su supuesto efecto perjudicial para el sector en sí mismo, e incluso puede pensarse -de manera marginalmente independiente a la coyuntura misma- como la expresión de una posición ideológica ligada a la no intervención estatal en la economía. Curiosamente, sin embargo, el signo distribución no es impugnado en relación con estas ideas. Pese a que no aparece con gran frecuencia en estos discursos, su valor no es discutido e incluso se observan intentos de absorberlo dentro del tópico del crecimiento concatenado (MA2).

En segundo lugar, en los discursos de CFK, encontramos que los signos campo y distribución, a través de sus relaciones con otros signos -rentabilidad, crecimiento, recuperación, producción-cobran su valor en el marco de la articulación de -al menos- dos tópicos, donde uno está subordinado a otro: (CF2) la necesidad de una política de redistribución del ingreso, se basa en (CF1) el mejoramiento de las condiciones macroeconómicas que, 
por acción del gobierno, generó excedente. En el caso de campo, su utilización subraya un rol del sector agropecuario en la recuperación económica como beneficiario y no como agente, mientras que, en relación con distribución, la fuerte relación de asociación que se establece con el signo rentabilidad señala un crecimiento "extraordinario" que puede y debe ser "distribuido". Esto último a la vez se combina con el lugar de privilegio que se le da a este programa de redistribución, al presentarlo como la respuesta a problemáticas que se consideran activas en el interdiscurso con independencia de la coyuntura del conflicto (preocupación por el problema de la pobreza, la distribución del ingreso como "tarea pendiente"). Por otra parte, las redefiniciones que se construyen a través de las referencias al interdiscurso y la puesta en discusión de posiciones marcadas como externas al propio discurso -en especial mediante la negación polémicapueden pensarse también como actualizaciones de los tópicos que se presentan, ya que establecen relaciones más o menos explícitas entre objetivos políticos y la coyuntura de la disputa en torno a las retenciones. En este caso, entonces, la lucha por la iniciativa discursiva parece encararse desde un poder hacer que legitima el poder decir -en este caso, poder definir (componente didáctico) y poder proponer (componente programático, Verón, 1987), en especial-.

El análisis de estos dos signos parece indicar la presencia de dos paradigmas de disputa en torno a los valores que se pretenden imponer a los signos estudiados, y que podemos relacionar con los tópicos analizados. Puede pensarse que el foco de la disputa es, en este caso, el rol del Estado en la generación de crecimiento económico y de la distribución equitativa de sus efectos. Podríamos denominar, entonces, paradigma del derrame, contrario a la intervención estatal, a lo relevado en los discursos de los dirigentes agrarios y paradigma intervencionista, que propone una política de regulación estatal, al correspondiente a los discursos presidenciales.

De esta manera, el signo campo muestra en cada configuración discursiva valores o acentuaciones diversas. Por una parte, en el caso de los dirigentes agrarios adquiere un rol privilegiado en la presentación del funcionamiento de la economía nacional, en tanto es lo que impulsa el modelo agroexportador, que genera -generó y generará- el "derrame económico" que asegura la bonanza general. Por otra parte, en el caso del discurso presidencial, campo es uno de los beneficiarios directos de la recuperación económica impulsada por las políticas del gobierno. En este marco, podemos afirmar que en el caso del signo campo la iniciativa discursiva no puede establecerse con claridad.

Por otra parte, el signo distribución se vuelve central para la construcción de un programa de gobierno en los discursos de CFK, en especial en el pasaje que va del tópico de la recuperación (CF1) al tópico de la nece- 
sidad de distribuir el excedente generado (CF2). Aquí, su relación con campo a través del signo rentabilidad es clave, dado que no existe distribución posible sin la "renta extraordinaria del campo", producida, según este discurso, por las condiciones favorables generadas por el Gobierno. Como se ha mencionado, el valor del signo distribución no es impugnado en los discursos de los dirigentes agrarios, que se ven relegados en este punto a una posición de respuesta. Por estas dos razones, la iniciativa discursiva parece pertenecer, en el caso de distribución y el tópico de la distribución del ingreso, al discurso presidencial.

Finalmente, es digno de remarcar que el análisis aquí presentado debe complementarse con el estudio de otros signos que se vuelven significativos en estos discursos, y que revelan la importancia de otros tópicos en juego en la polémica discursiva abordada. 


\section{BIBLIOGRAFÍA}

Adelstein, A. y Vommaro, G. (eds.) (2014): Diccionario del léxico corriente de la politica argentina. Palabras en democracia (1983-2013), Los Polvorines, Universidad Nacional de General Sarmiento.

AlbeldA, M. (2005): La intensificación en el español coloquial, Valencia, Universidad de Valencia.

Angenot, M. (2010): El discurso social. Los límites históricos de lo pensable y de lo decible, Buenos Aires, Siglo XXI.

Aronskind, R. (2014): “Campo", en A. Adelstein y G. Vommaro (eds.), Diccionario del léxico corriente de la política argentina. Palabras en democracia (1983-2013), Los Polvorines, Universidad Nacional de General Sarmiento, págs. 69-72.

Authier-Revuz, J. (1984): "Hetérogénéité(s) énonciative(s)", Langages, 73, págs. 98-111.

Blas Arroyo, J. L. (2010): "La función de las preguntas en un discurso agonal: el debate electoral cara a cara", Discurso \& Sociedad, 4 (4), págs. 674-705.

Chouliaraki, L. y Fairclough, N. (2001): Discourse in Late Modernity, Edimburgo, Edinburgh University Press.

Dubois, J. (1962): Le vocabulaire politique et social en France de 1869 à 1872, París, Larousse.

Dvoskin, G. (2013): "Paradigmas en disputa, presupuestos compartidos", Revista Aled, 13 (1), págs. 79-98.

Fairclough, N. (1995): Media Discourse, Londres, Edward Arnold.

Foucault, M. (1971): El orden del discurso, Tusquets, Barcelona.

García Negroni, M. M. (2009): "Negación y descalificación: a propósito de la negación metalingüística”, Ciências \& Letras, 45, págs. 61-82.

GASPARINI, L. (2006): "La distribución del ingreso: un breve resumen de tres décadas”, Económica, 52 (1), págs. 53-74.

Gaviola, S. R. y Anchorena, S. O. (2008): “¿Qué es lo que derrama el efecto derrame?”, en AA.VV., Actas de las Segundas Jornadas de Economía Política, General Sarmiento, Universidad Nacional de General Sarmiento.

Lakoff, G. y Johnson, M. (1998): Metáforas de la vida cotidiana, Madrid, Cátedra.

Lissin, L. (2010): Federación Agraria hoy. El campo argentino en discusión, Buenos Aires, Capital Intelectual.

Maingueneau, D. (1994): Nuevas tendencias en análisis del discurso, Madrid, Hachette.

Palma, A. G. (2011): "Funciones estratégicas y construcción de destinatarios durante el conflicto agrario argentino del año 2008: el caso de los pronombres personales en el discurso de Alfredo de Angeli”, Revista Interdisciplinaria de Estudios Sociales, 3, págs. 61-96.

- (2013): "Cuando nombrar es clasificar: paradigmas de disputa en torno al conflicto agrario argentino del año 2008. El caso de los periódicos Clarín y 
Página 12", en F. G. Romero Wimer et al., El complejo agroalimentario pampeano 1976-2012: Estructura económica, dinámica política y trayectorias sociales, Bahía Blanca, Ediciones del Ceiso, págs. 131-182.

- (2014): "Quienes protestan y por qué: construcción de destinatarios durante el conflicto en torno a las retenciones móviles en la argentina (marzo-julio 2008)", Tonos Digital, 2 (27). <http://www.tonosdigital.com/ojs/index.php/ tonos/article/view/1150>.

PÊCHeux, M. (1988 [1975]): Semântica e discurso. Uma crítica à afirmacão do obvio, Campinas, San Pablo, Editora da Unicamp.

PÉrez, S. (1999): "Voces en el Parlamento", en A. Raiter et al., Discurso y ciencia social, Buenos Aires, Eudeba, págs. 135-141.

RAITER, A. (2009): "Hablo y entiendan: creencias, presuposición e interdiscurso en los actos de Cristina Fernández de Kirchner”, Oralia, 12, págs. 73-96.

- (2014): "Guiar la lectura del pasado: estructuras topológicas en CFK", en S. Pérez (ed.), Análisis del discurso político, Mendoza, Editorial FFyL-UNCuyo y SAL, págs. 77-88.

- et al. (1999): Lingüistica y política, Buenos Aires, Biblos.

SARTElli, E. (2008): Patrones en la ruta. El conflicto agrario y los enfrentamientos en el seno de la burguesía. Marzo-julio de 2008, Buenos Aires, Razón y Revolución.

TREW, T. (1983): "Lo que dicen los periódicos, variación lingüística y diferencia ideológica”, en R. Fowler (comp.), Lenguaje y control, México, FCE, págs. 159211.

VAn DijK, T. (1978): La ciencia del texto, Barcelona, Paidós.

- (comp.) (2000): El discurso como interacción social. Estudios sobre el discurso, II. Una introducción multidisciplinaria, Barcelona, Gedisa.

Verón, E. (1987): "La palabra adversativa", en E. Verón et al., El discurso político: lenguajes y acontecimientos, Buenos Aires, Hachette, págs. 13-26.

Voloshinov, V. (1976 [1926]): El marxismo y la filosofia del lenguaje, Buenos Aires, Nueva Visión. 


\section{ANEXO}

CONVENCIONES DE TRANSCRIPCIÓN

\begin{tabular}{|l|l|}
\hline$::::$ & Alargamiento de la sílaba previa. \\
\hline MAYÚSCULAS: & $\begin{array}{l}\text { Mayor volumen de emisión o fragmentos } \\
\text { de emisión. }\end{array}$ \\
\hline$\underline{\text { Subrayado: }}$ & Énfasis mayor en la emisión del hablante. \\
\hline Punto entre paréntesis $():$. & Pausa de una décima de segundo. \\
\hline Números entre paréntesis $(1.7):$ & Segundos y décimas de segundos de pausa. \\
\hline ?: Entonación ascendente: & Entonación interrogativa. \\
\hline ((Doble paréntesis) $):$ & Aclaraciones, descripciones. \\
\hline Paréntesis incluyendo una x: $(x)$ & $\begin{array}{l}\text { Detención repentina o vacilación } \\
\text { por parte del hablante. }\end{array}$ \\
\hline
\end{tabular}

